\title{
Tuning of the Parameters of PSS and IPFC-POD Controllers Using the Current Sensitivity Model
}

\author{
Elenilson de Vargas Fortes*. Leonardo H. Macedo**. Percival Bueno de Araujo** \\ Ednei Luiz Miotto**** \\ *Federal Institute of Goias, Jatai-GO, CEP 75.804-020, Brazil \\ (Tel: (64)3605-0800; e-mail: elenilson.fortes@ifg.edu.br) \\ **São Paulo State University, Ilha Solteira-SP, CEP 15.385-000, Brazil \\ (Tel: (18)3743-1000; e-mail: leohfmp@ieee.org and percival@dee.feis.unesp.br). \\ ***Federal Technological University of Paraná, Toledo-PR, CEP 85902-490, Brazil \\ (Tel: (45) 3379-6800; e-mail: edneimiotto@utfpr.edu.br).
}

\begin{abstract}
This paper presents a proposal for a coordinated adjustment of the parameters of supplementary damping controllers (Power System Stabilizers and Interline Power Flow Controller (IPFC)-Power Oscillation Damping (POD)) using the Multi-Start algorithm. The aim is to introduce additional damping to electromechanical oscillations present in electric power systems (EPSs). The current sensitivity model (CSM), is used to represent the EPS. The fundamental concept of the CSM is the nodal balance of currents at each time instant and in each bus of the power system. Thus, all devices and components of the EPS are modelled by current injection, including the IPFC. The solution is applied to a test system with 4 generators, 10 buses and 15 transmission lines divided into two areas with the presence of local and inter-area mode oscillations. The efficiency of the Multi-Start algorithm in adjusting the parameters of controllers can be concluded from the results.
\end{abstract}

Resumo: Este artigo apresenta uma proposta para o ajuste coordenado dos parâmetros dos controladores suplementares de amortecimento (Estabilizadores do Sistema de Potência (ESP) e Interline Power Flow Controller (IPFC) - Power Oscillation Damping (POD)) usando o algoritmo Multi-Start. O objetivo é introduzir amortecimento adicional às oscilações eletromecânicas presentes nos sistemas elétricos de potência (SEP). O modelo de sensibilidade atual (MSC) é usado para representar o SEP. O conceito fundamental do MSC é baseado no balanço nodal das correntes em cada instante de tempo e em cada barra do sistema de potência. Assim, todos os dispositivos e componentes do SEP são modelados por injeção de corrente, incluindo o IPFC. A proposta é aplicada a um sistema teste com 4 geradores, 10 barras e 15 linhas de transmissão divididos em duas áreas e com a presença de oscilações de modo local e interárea. A eficiência do algoritmo Multi-Start no ajuste dos parâmetros dos controladores pôde ser concluída a partir dos resultados.

Keywords: Current sensitivity model; interline power flow controller; Multi-Start algorithm; power oscillation damping; power system stabilizers

Palavras-chaves: Modelo de sensibilidade de corrente; interline power flow controller, algoritmo Multi-Start; power oscillation damping; estabilizadores de sistema de potência.

\section{INTRODUCTION}

The stability of EPSs is related to their capacity to remain in equilibrium under normal operating conditions. Furthermore, the EPS must evolve to an operating state within established standards when subjected to disturbances, which can be of small or large magnitudes. The study of the behavior of EPSs with small normal load variations in buses is called small signal stability Anderson and Fouad (1993).

The disturbances present in an EPS can cause low-frequency electromechanical oscillations that, depending on the magnitude, can make it unstable. In view of this, control strategies to introduce additional damping to these oscillations should be applied. Oscillations are classified as local $(0.8$ to $2.0 \mathrm{~Hz})$, inter-area $(0.2$ to $0.7 \mathrm{~Hz}$ ) or intraplant $(1.5$ to $2.5 \mathrm{~Hz})$ and are identified from their frequency of occurrence Anderson and Fouad (1993); De Mello and Concordia (1969); Larsen and Swann (1981). Local and inter-area oscillations are of interest in this work.

The first steps to introduce damping to these oscillations were presented in De Mello and Concordia (1969), with this issue being taken up again in Larsen and Swann (1981). In these studies, the Power System Stabilizer controller (PSS) was added to the control loop of Automatic Voltage Regulators (AVRs) with the function of producing electrical torque in phase with the variations of the angular velocity of the rotor (damping torque). From these studies, the authors concluded that when the parameters (time and gain constants) of this device are well adjusted, it is possible to provide additional damping to local mode oscillations 
De Mello and Concordia (1969); Larsen and Swann (1981). However, the PSS is not efficient to dampen inter-area mode oscillations and, depending on the settings of the parameters, it can negatively influence the damping of these oscillations.

With the development of power electronics, new automatic compensation devices have been incorporated into EPSs, including the Flexible AC Transmission System (FACTS) technology Hingorani and Gyugyi (1999). These devices have made the operation of EPSs less rigid, increasing the capability of power transfer in existing systems, with continuous control of power flow at specific locations of the transmission system and providing significant improvements in voltage levels. The FACTS devices do not have the ability to introduce damping to the oscillations of the EPS. However, if a Power Oscillation Damping (POD) controller is added to its control loop, the FACTS-POD pair can provide additional damping to inter-area oscillations Valle and Araujo (2015); Wang et al. (2007).

The adjustment of the parameters of the PSSs and the Interline Power Flow Controller (IPFC)-POD is of fundamental importance to introduce damping to the different oscillation modes of the EPS. Methods based on classic control have been used to set these parameters Anderson and Fouad (1993); De Mello and Concordia (1969); Larsen and Swann (1981). More recently, optimization methods, for example, the, Particle Swarm Optimization Wang et al. (2007), the Chu-Beasley's Genetic Algorithm Fortes et al. (2016a), Simulated Annealing Fortes et al. (2016b), Variable Neighborhood Search Fortes et al. (2018), the Fireworks Algorithm Fortes et al. (2017) and the Artificial Bee Colony Algorithm Martins et al. (2017), have also been used.

This paper investigates the joint action of the IPFC FACTS Gyugyi et al. (1999) and the supplementary controller POD in damping low-frequency inter-area electromechanical oscillations. It is proposed to apply an optimization method based on the Multi-Start algorithm Glover and Kochenberger (2003) to coordinate adjustments of parameters of supplementary damping controllers (PSSs and IPFC-POD) and thus insert additional damping to the EPS (which is represented by the CSM) Takahashi et al. (2018).

In this context, the main contributions of this work are employing the Multi-Start algorithm to perform coordinated adjustments of the parameters of PSSs and IPFC-POD and represent the IPFC by current injection.

\section{CURRENT SENSITIVITY MODEL}

The CSM is a linear analysis tool for EPSs with its central concept based on Kirchhoff's current law. The current balance must be met at all nodes for each and every dynamic process in the EPS and it is applied to obtain the algebraic equations of the CSM Takahashi et al. (2018).

In this model, the state variables are represented by $\Delta x$ (angular velocity of the synchronous generator $(\Delta \omega)$, the internal rotor angle of the generator $(\Delta \delta)$, quadrature-axis component of the internal voltage $\left(\Delta E_{q}^{\prime}\right)$ and the field voltage of the generator $\left(\Delta E_{f d}\right)$ ), the input by $\Delta u$ (the mechanical power, $\Delta P_{m}$, and the reference voltage $\left(\Delta V_{\text {ref }}\right)$ of the AVR of each generator) and the algebraic equations by $\Delta z$ (magnitude $(\Delta V)$ and phase angle $(\Delta \theta)$ of the voltage of each EPS bus).
A multimachine power system, composed of $n g$ generators and $n b$ buses, can be represented by the CSM in the time domain, as shown in (1)-(4).

$$
\begin{aligned}
& \Delta x=\left[\left[\Delta \omega_{1} \cdots \Delta \omega_{n g}\right]\left[\Delta \delta_{1} \cdots \Delta \delta_{n g}\right]\left[\Delta E_{q_{1}}^{\prime} \cdots \Delta E_{q_{n g}}^{\prime}\right]\right. \\
& \left.\left[\Delta E_{f d_{1}} \cdots \Delta E_{f d_{n g}}\right]\right]^{t} \\
& \Delta u=\left[\left[\Delta P_{m_{1}} \cdots \Delta P_{m_{n g}}\right]\left[\Delta V_{r e f_{1}} \cdots \Delta V_{r e f_{n g}}\right]\right]^{t} \\
& \Delta z=\left[\left[\Delta \theta_{1} \cdots \Delta \theta_{n b}\right]\left[\Delta V_{1} \cdots \Delta V_{n b}\right]\right]^{t} \\
& {\left[\begin{array}{c}
\Delta \dot{x} \\
0
\end{array}\right]=\left[\begin{array}{cc}
J_{1} & J_{2} \\
J_{3} & J_{4}
\end{array}\right]\left[\begin{array}{c}
\Delta x \\
\Delta z
\end{array}\right]+\left[\begin{array}{c}
B_{1} \\
B_{2}
\end{array}\right] \Delta u}
\end{aligned}
$$

Additional information about (1)-(4) can be obtained from Takahashi et al. (2018).

\section{INTERLINE POWER FLOW CONTROLLER}

Generally speaking, the IPFC can manage and control power flows in the substation where it is installed and thus can reduce transmission power overloads and improve the voltage profile in EPS buses Hingorani and Gyugyi (1999); Valle and Araujo (2015); Gyugyi et al. (1999).

To obtain a mathematical model that represents the IPFC, consider Fig. 1 which shows the equivalent circuit of the device.

Active and reactive power injections at bus $i$ are represented by $P_{i}$ and $Q_{i} . P_{j i}, P_{k i}, Q_{j i}$ and $Q_{k i}$ are the active and reactive power flows in the lines in which the IPFC is installed (leaving buses $j$ and $k$ ), respectively. $\boldsymbol{I}_{i j}$ and $\boldsymbol{I}_{i k}$ designate the currents that leave bus $i$, while $\boldsymbol{I}_{j i}$ and $\boldsymbol{I}_{k i}$ represent the currents leaving buses $j$ and $k$, respectively. Assuming that active losses are zero, it follows that the active power provided by one of the converters (VSC1) will always be equal to the active power demand of the other (VSC2), resulting in (5) (active power invariance of the IPFC Zhang (2003)).

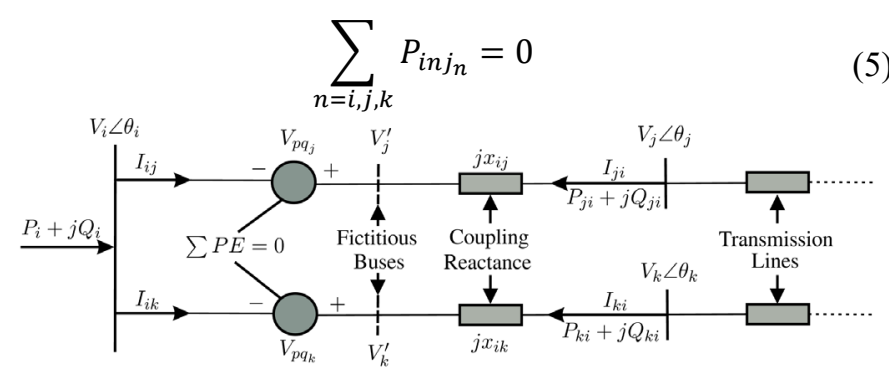

Figure 1. Equivalent circuit of an IPFC with two series voltage sources.

The control of power flow in the line in which the IPFC is installed is achieved by modulating the magnitude and angle of the series voltage sources $V_{p q_{n}},(n=j, k)$, as shown in Fig. 1 and that can be expressed as (6).

$$
V_{p q_{n}}=r_{n} e^{j \gamma_{n}} \boldsymbol{V}_{i}
$$

Moreover, $0<\gamma_{n}<2 \pi$ and $0<r_{n}<r_{n}^{\max }$ are parameters of the series voltage sources, where $r_{n}=V_{p q_{n}} / \boldsymbol{V}_{i}$ and $\gamma_{n}$ is the angle of $V_{p q_{n}}$ in relation to the voltage in bus $\boldsymbol{V}_{i}$ Zhang and Yokoyama (2008). 
The controllable voltage, $V_{p q_{n}}$, can be decomposed into one component in phase $\left(V_{q n}\right)$ and another in quadrature $\left(V_{p n}\right)$, as in (7) and (8) (see Fig. 2).

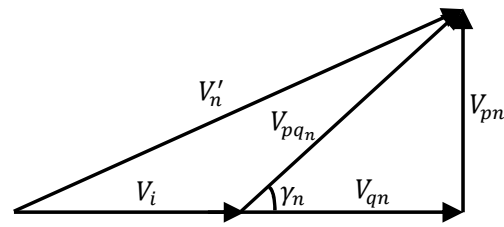

Figure 2. Phasor diagram of the IPFC.

$$
\begin{aligned}
& V_{q n}=r_{n} \boldsymbol{V}_{i} \cos \gamma_{n} \\
& V_{p n}=r_{n} \boldsymbol{V}_{i} \sin \gamma_{n}
\end{aligned}
$$

From the equivalent circuit of the IPFC (Fig. 1) and considering the constraints in (5)-(8), it is possible to deduce the power injection model for the IPFC, (9)-(12). The active and reactive power injections at bus $i$ are $P_{i}$ and $Q_{i}$, while $P_{n i}$ and $Q_{n i}$ represent of active and reactive power injections in buses $j$ and $k$.

$$
\begin{aligned}
& P_{i}=-\sum_{n=j, k} V_{i} V_{n} b_{i n} \sin \theta_{i n}-\underbrace{V_{i} \sum_{n=j, k} b_{i n} V_{p n}}_{\operatorname{IPFC}\left(P_{i n j_{i}}\right)} \\
& Q_{i}=-V_{i}^{2} b_{i i}+\sum_{n=j, k} V_{i} V_{n} b_{i n} \cos \theta_{i n}-\underbrace{\sum_{n=j, k} b_{i n} V_{q n}}_{\operatorname{IPFC}\left(Q_{i n j_{i}}\right)} \\
& P_{n i}=V_{i} V_{n} b_{i n} \sin \theta_{i n}+\underbrace{V_{n} b_{i n}\left(V_{q n} \sin \theta_{i n}+V_{p n} \cos \theta_{i n}\right)}_{\operatorname{IPFC}\left(P_{i n j_{n}}\right)} \\
& Q_{n i}=-V_{i}^{2} b_{i n}+V_{i} V_{n} b_{i n} \cos \underbrace{\cos +V_{\text {in }}\left(V_{q n} \cos \theta_{i n}-V_{p n} \sin \theta_{i n}\right)}_{\text {in }}
\end{aligned}
$$

In (9)-(12), $\theta_{\text {in }}=\theta_{i}-\theta_{n}, b_{\text {in }}=-1 / x_{\text {in }}$ ( $x_{\text {in }}$ is the reactance of the coupling transformers), and $b_{i n}=\sum_{n=j, k} 1 / x_{i n}$. Note that these equations are not dependent on the parameters $r_{n}$ and $\gamma_{n}$ present in (7) and (8), thereby eliminating the need for their calculations.

The underlined part with the description "IPFC", refers to the contribution of the device in the power flow of the transmission line it is installed. Thus, these contributions are power injections by the device to the buses it is connected $\left(P_{i n j_{i}}, Q_{i n j_{i}}, P_{i n j_{n}}\right.$, and $Q_{i n j_{n}}$, for $\left.n=j, k\right)$. This makes it possible to represent the IPFC by a power injection model (Fig. 3).

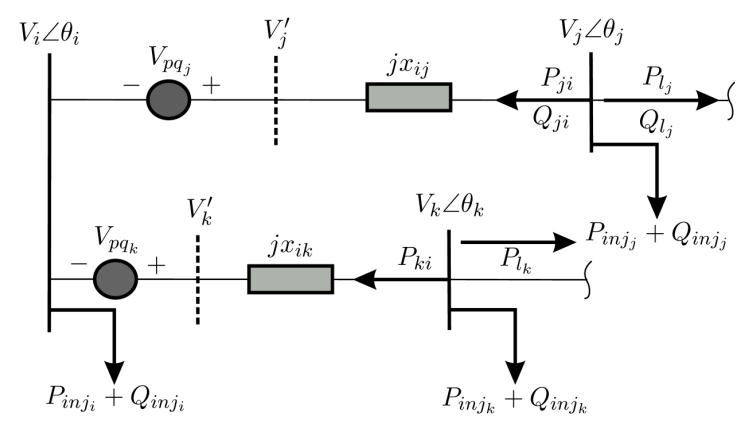

Figure 3. Power injection model and flow control by the IPFC.
Note that the model shown in Fig. 3 facilitates the inclusion of an IPFC in a power flow algorithm. The equations of conventional power flow for the buses without the device are kept and the contributions of the IPFC are considered as if they are injections in the buses of its installation. The balances of active and reactive power in the buses where the IPFC is connected are given by (13) and (14), respectively.

$$
\begin{gathered}
P_{g m}-P_{i n j_{m}}-P_{l_{m}}-P_{c a l_{m}}=0 \\
Q_{g m}-Q_{i n j_{m}}-Q_{l_{m}}-Q_{c a l_{m}}=0
\end{gathered}
$$

In (13) and (14), $m=i, j$ and $k$. The active and reactive powers generated are $P_{g m}$ and $Q_{g m}$ (entering bus $m$ ). Possible loads drain from bus $m$ active and reactive powers $\left(P_{l_{m}}\right.$ and $Q_{l_{m}}$, respectively). $P_{c a l_{m}}$ and $Q_{c a l_{m}}$ are the conventional active and reactive powers transmitted by the transmission lines (leaving bus $m$ ). The injections of active and reactive power by the IPFC are $P_{i n j_{m}}$ and $Q_{i n j_{m}}$.

The control of the power flows $P_{l_{j}}, P_{l_{k}}$ (active) and $Q_{l_{j}}$ (reactive) is expressed by (15)-(17).

$$
\begin{aligned}
& P_{l_{j}}-P_{\text {ref }}=0 \\
& P_{l_{k}}-P_{\text {ref }_{k}}=0 \\
& Q_{l_{j}}-Q_{\text {ref }}=0
\end{aligned}
$$

In (15)-(17) the values specified for the active and reactive power flows for transmission line $i-j$ are $P_{r e f_{j}}$ and $Q_{r e f_{j}} . P_{r e f_{k}}$ is the value specified for the active power flow in the line $i-k$.

Proportional-Integral controllers (PI) are used to control the flows as shown in Fig. 4 Valle and Araujo (2015). Note that the controllers modulate the variables of controllable sources, except for the variable $V_{q_{k}}$, which is related to the equation of active power invariance (5).

In the structure shown in Fig. 4 , the time constant, $T_{m_{p}}$, in the interval 1-10 ms, represents the inherent delay of the control device Hingorani and Gyugyi (1999). The parameters of the PI controllers are the gains, $K 1, K 2$, and $K 3$ and the time constants, $T_{1_{i}}$, $T_{2_{i}}$ and $T_{3_{i}}$. The signal $V_{\text {sup }}$ is from a POD and in this work, it will be used to modulate the variable $V_{p_{j}}$, and thus provide additional damping to the electromechanical oscillations of the EPS.
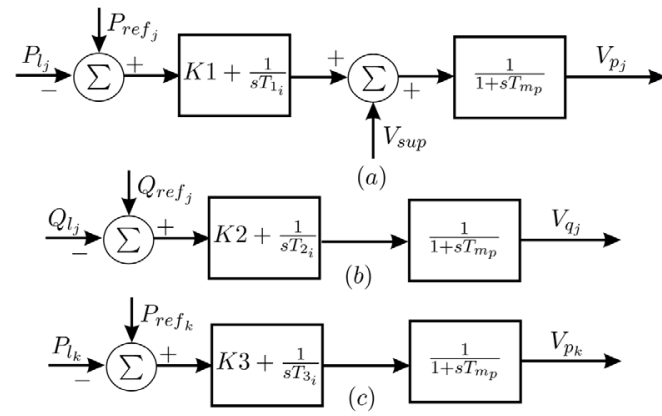

Figure 4. Structure of the control system of the IPFC.

The dynamic behavior of the control structure, obtained by inspection, is shown in Fig. 4 and is given by (18)-(23). 
$\dot{V}_{p_{j}}=\frac{K 1}{T_{m_{p}}}\left(P_{\text {ref }_{j}}-P_{l_{j}}\right)+\frac{1}{T_{m_{p}}} X_{1}+\frac{1}{T_{m_{p}}} V_{\text {sup }}-\frac{1}{T_{m_{p}}} V_{p_{j}}$

$\dot{X}_{1}=\frac{1}{T_{1_{i}}}\left(P_{r e f_{j}}-P_{l_{j}}\right)$

$\dot{V}_{q_{j}}=\frac{K 2}{T_{m_{p}}}\left(Q_{r e f_{j}}-Q_{l_{j}}\right)+\frac{1}{T_{m_{p}}} X_{2}-\frac{1}{T_{m_{p}}} V_{q_{j}}$

$\dot{X}_{2}=\frac{1}{T_{2_{i}}}\left(Q_{r e f_{j}}-Q_{l_{j}}\right)$

$\dot{V}_{p_{k}}=\frac{K 3}{T_{m_{p}}}\left(P_{r e f_{k}}-P_{l_{k}}\right)+\frac{1}{T_{m_{p}}} X_{3}-\frac{1}{T_{m_{p}}} V_{k}$

$\dot{X}_{3}=\frac{1}{T_{3_{i}}}\left(P_{r e f_{k}}-P_{l_{k}}\right)$

\section{CURRENT INJECTION MODEL FOR THE IPFC}

The current injection model for the IPFC that will be employed (one of the contributions of this work), is obtained from the equivalent circuit shown in Fig. 1. Unlike the equations reported in Rezaei et al. (2013), the currents injected by the device are calculated considering controllable sources with the appropriate reactances of the coupling transformers. Thus, (24) and (25) follow from Fig. 1.

$$
\begin{gathered}
\boldsymbol{I}_{i}=\boldsymbol{I}_{i j}+\boldsymbol{I}_{i k}=-\left(V_{i} \angle \theta_{i}+r_{j} e^{j \gamma_{j}} V_{i}-V_{j} \angle \theta_{j}\right) / x_{i j} \\
-\left(V_{i} \angle \theta_{i}+r_{k} e^{j \gamma_{k}} V_{i}-V_{k} \angle \theta_{k}\right) / x_{i k} \\
\boldsymbol{I}_{n i}=\left(V_{n}-V_{n}^{\prime}\right) / j x_{i n} \\
=-\left(V_{n} \angle \theta_{n}-V_{i} \angle \theta_{i}-r_{n} e^{j \gamma_{n}} V_{i} \angle \theta_{i}\right) / x_{i n}
\end{gathered}
$$

Equations (26) and (27) are determined by substituting (7) and (8) in (24) and (25).

$$
\begin{gathered}
\boldsymbol{I}_{i}=V_{i} \sin \theta_{i}\left(-b_{i j}-b_{i k}\right)+b_{i j} V_{j} \cos \theta_{j}+b_{i k} V_{k} \sin \theta_{k} \\
-\sum_{n=j, k} b_{i n}\left(V_{p_{n}} \cos \theta_{i}+V_{q_{n}} \sin \theta_{i}\right)+ \\
j\left[-V_{i} \cos \theta_{i}\left(-b_{i j}-b_{i k}\right)-b_{i j} V_{j} \cos \theta_{j}-b_{i k} V_{k} \cos \theta_{k}\right. \\
\left.+\sum_{n=j, k} b_{i n}\left(V_{q_{n}} \cos \theta_{i}-V_{p_{n}} \sin \theta_{i}\right)\right] \\
\boldsymbol{I}_{n i}=b_{i n}\left(V_{i} \sin \theta_{i}-V_{n} \sin \theta_{n}\right)+ \\
b_{i n}\left(V_{p_{n}} \cos \theta_{i}+V_{q_{n}} \sin \theta_{i}\right)+ \\
j\left[b_{i n}\left(V_{n} \cos \theta_{n}-V_{i} \cos \theta_{i}\right)\right. \\
\left.+b_{i n}\left(V_{p_{n}} \sin \theta_{i}-V_{q_{n}} \cos \theta_{i}\right)\right]
\end{gathered}
$$

In (26) and (27), $b_{i n}=-1 / x_{i n}$ and $n=j, k$. These can be separated into their real and imaginary components as in (28)-(31).

$$
\begin{array}{r}
I_{i_{r}}=V_{i} \sin \theta_{i}\left(-b_{i j}-b_{i k}\right)+b_{i j} V_{j} \cos \theta_{j}+b_{i k} V_{k} \sin \theta_{k} \\
-\underbrace{\sum_{n=j, k} b_{i n}\left(V_{p_{n}} \cos \theta_{i}+V_{q_{n}} \sin \theta_{i}\right)}_{\operatorname{IPFC}\left(I_{i n j_{i r}}\right)}
\end{array}
$$

$$
\begin{gathered}
I_{i_{m}}=-V_{i} \cos \theta_{i}\left(-b_{i j}-b_{i k}\right)-b_{i j} V_{j} \cos \theta_{j}-b_{i k} V_{k} \cos \theta_{k} \\
+\underbrace{\sum_{n=j, k} b_{i n}\left(V_{q_{n}} \cos \theta_{i}-V_{p_{n}} \sin \theta_{i}\right)}_{\operatorname{IPFC}\left(I_{i n j_{i_{m}}}\right)} \\
I_{n i_{r}}=b_{i n}\left(V_{i} \sin \theta_{i}-V_{n} \begin{array}{r}
\left.\sin \theta_{n}\right) \\
+\underbrace{b_{i n}\left(V_{p_{n}} \cos \theta_{i}+V_{q_{n}} \sin \theta_{i}\right)}_{\operatorname{IPFC}\left(I_{i n j_{n}}\right)}
\end{array}\right. \\
I_{n i_{m}}=b_{i n}\left(V_{n} \cos \theta_{n}-V_{i} \cos \theta_{i}\right) \\
+\underbrace{b_{i n}\left(V_{p_{n}} \sin \theta_{i}-V_{q_{n}} \cos \theta_{i}\right)}_{\operatorname{IPFC}\left(I_{i n j_{n_{m}}}\right)}
\end{gathered}
$$

In (28)-(31), the underlined part with "IPFC" refers to the contribution to current flow by the device where it is installed. Current injections at bus $i$ are $I_{i n j_{i_{r}}}$ and $I_{i n j_{i_{m}}} . I_{i n j_{n_{r}}}$ and $I_{i n j_{n_{m}}}$ are the currents injected into bus $n(n=j, k)$ of the real and imaginary components, as shown in Fig. 5.

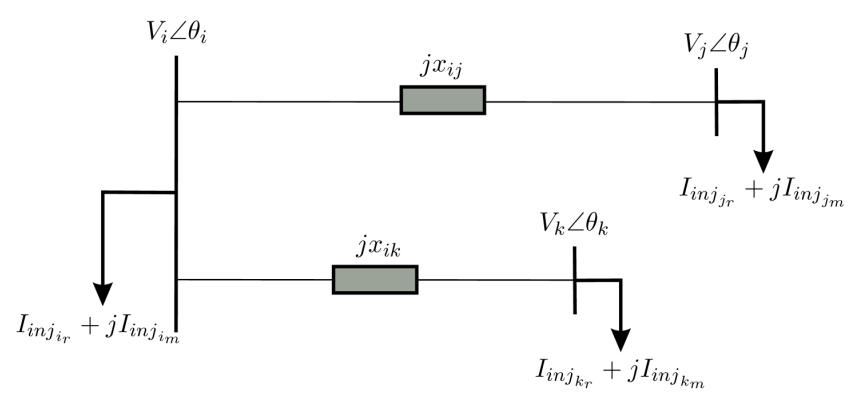

Figure 5. Current injection model for the IPFC.

\section{STRUCTURES OF THE PSS AND POD CONTROLLERS}

To introduce damping to the oscillations of the EPS, it is necessary to include supplementary damping controllers (PSSs and POD). In this work, it is assumed that the structures of these controllers are the same (Fig. 6) differing only in input and output signals. The input signal $\Delta V_{i n}$ chosen for the PSSs is $\Delta \omega_{k}$ (variations of the angular velocity of the rotor of generator $k$ ). The input adopted for the POD is $\Delta P_{k m}$ (deviations of the active power flow in the transmission line adjacent to the installation of the IPFC-POD). The output of the PSS, $\Delta V_{\text {sup }}$, is applied to the generator voltage control loop. The output of the POD, $\Delta V_{\text {sup }_{2}}$, is added to the control loop of the IPFC device (Fig. 7).

In Fig. 7(a), the AVR is represented by a gain, $K_{r_{k}}$, and a time constant, $T_{r_{k}}$. The generator field voltage is $E_{f d_{k}}$, the magnitude of the terminal voltage is $V_{k}$, and the reference voltage is $V_{r e f_{k}}$. In Fig. 7(b), the POD is coupled to the control loop of the IPFC to modulate $\Delta V_{p_{j}} . X_{\text {ref }}$ is the input signal, and $T_{m_{p}}$ is the time constant both of which are specified.

The equations that define the behavior of PSSs, (32)-(35), and POD, (36)-(39), are obtained by analyzing the block diagrams shown in Figs. 6 and 7. 




Figure 6. The dynamic model of the PSSs and POD controller.

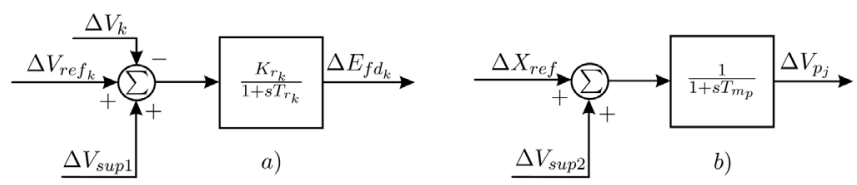

Figure 7. Dynamic model of the PSSs and POD: (a) Control loop of the AVRs (b) Quadrature voltage component of the primary converter of the IPFC.

$$
\begin{aligned}
& \Delta \dot{V}_{1_{k}}=\Delta \dot{\omega}_{k} K_{P S S}-\frac{1}{T_{\omega}} \Delta V_{1_{k}} \\
& \Delta \dot{V}_{2_{k}}=\frac{1}{T_{2}} \Delta V_{1_{k}}+\frac{T_{1}}{T_{2}} \Delta \dot{V}_{1_{k}}-\frac{1}{T_{2}} \Delta V_{2_{k}} \\
& \Delta \dot{V}_{\text {sup }_{1}}=\frac{1}{T_{4}} \Delta V_{2_{k}}+\frac{T_{3}}{T_{4}} \Delta \dot{V}_{2_{k}}-\frac{1}{T_{4}} \Delta V_{s u p_{1}} \\
& \Delta \dot{E}_{f d_{k}}=\frac{K_{r}}{T_{r}} \Delta V_{s u p_{1}}+\frac{K_{r}}{T_{r}} \Delta V_{r e f_{k}}-\frac{K_{r}}{T_{r}} \Delta V_{k}-\frac{1}{T_{r}} \Delta E_{f d_{k}} \\
& \Delta \dot{Y}_{1}=\frac{1}{T_{\omega_{p}}}\left(K_{P O D} \Delta P_{k m}-\Delta Y_{1}\right) \\
& \Delta \dot{Y}_{2}=\frac{1}{T_{p_{2}}}\left[\left(1-\frac{T_{p_{1}}}{T_{p_{2}}}\right)\left(K_{P O D} \Delta P_{k m}-\Delta Y_{1}\right)-\Delta Y_{2}\right] \\
& \Delta \dot{Y}_{3}=\frac{1}{T_{p_{4}}}\left[\Delta Y_{2}+\frac{T_{p_{1}}}{T_{p_{2}}}\left(K_{P O D} \Delta P_{k m}-\Delta Y_{1}\right)\left(1-\frac{T_{p_{3}}}{T_{p_{4}}}\right)-\Delta Y_{3}\right] \\
& \Delta V_{\text {sup }_{2}}=\Delta Y_{3}+\frac{T_{p_{3}}}{T_{p_{4}}}\left[\Delta Y_{2}+\frac{T_{p_{1}}}{T_{p_{2}}}\left(K_{P O D} \Delta P_{k m}-\Delta Y_{1}\right)\right]
\end{aligned}
$$

In (32)-(39), time constants, $T_{1}$ to $T_{4}$ and $T_{p_{1}}$ to $T_{p_{4}}$, and the gains, $K_{P S S}$ and $K_{P O D}$, are the parameters of the PSSs and POD, respectively. In the literature, it is usual to adopt $T_{1}=T_{3}, T_{2}=$ $T_{4}, T_{p_{1}}=T_{p_{3}}$ and $T_{p_{2}}=T_{p_{4}}$, Kundur (1994).

\section{ALGORITHM FOR SETTING PARAMETERS}

With the EPS modeled by the CSM, it is possible to assess its static behavior (voltage profile) and its dynamic behavior (stability). The voltage profile is evaluated by means of a power flow algorithm, and stability by analyzing the eigenvalues of the state matrix (which can be determined from (4) Takahashi et al. (2018)). If any problem in the behavior of the EPS is identified, actions must be taken to solve it, either to improve the voltage profile or to insert damping to local and inter-area mode oscillations. In the case of this work, the actions are to install PSSs and the IPFC-POD. The problem then is to adjust the parameters of these controllers to obtain the desired performance.

For this, consider an EPS equipped with $n$ PSSs in $n$ synchronous machines and an IPFC-POD. Also, assume that the time constant of the washout block is specified at 1 second for the PSSs $\left(T_{\omega}\right)$ and 1 second for the IPFC-POD $\left(T_{\omega_{p}}\right)$. Then only the gains and time constants shown in Fig. 6 need to be determined. The representation of a proposed solution to the problem is given in Fig.8.

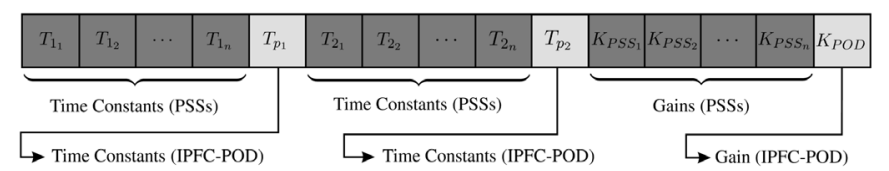

Figure 8. Representation of a proposal solution.

Note that the first $n$ positions of the representation of a proposal solution are destined to $n$ time constants $T_{1}$ of $n$ PSSs. The position $n+1$ refers to the time constant $T_{p 1}$ of the IPFC-POD. Keeping this order, $n$ time constants $T_{2}$ and then $T_{p 2}$, are represented. At the end, the $n$ gains $K_{P S S}$ (of the PSSs) and the gain $K_{P O D}$ (of the IPFC-POD) are represented. Each one of these variables is subject to its respective constraint (40)-(42).

$$
\begin{gathered}
1.00 \leq K_{E S P} \leq 5.00,0.05 \leq K_{P O D} \leq 1.00 \\
0.05 \leq T_{1_{n}} \leq 1.00,0.05 \leq T_{2_{n}} \leq 1.00 \\
0.05 \leq T_{p_{1}} \leq 1.00,0.05 \leq T_{p_{2}} \leq 1.00
\end{gathered}
$$

In the proposed approach, the aim is to solve a constraint satisfaction problem Glover and Kochenberger (2003) in which the desired damping is considered as a constraint of the problem, and any adjustment that provides the desired damping is considered a solution.

\subsection{Multi-Start Algorithm}

The Multi-Start algorithm Glover and Kochenberger (2003); Fortes et al. (2016b), as a strategy to leave local optimal solutions, is shown in Fig. 9.

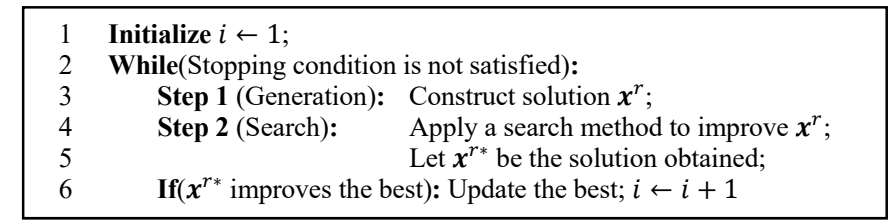

Figure 9. Multi-Start Algorithm Glover and Kochenberger (2003).

The Multi-Start algorithm of Fig. 9 consists of two steps, the first is where a solution is built and the second, a local search. These two steps should be repeated until the stop criterion is satisfied. In the proposal of this study, in Step 1, the solution is generated randomly for the first iteration $(i=1)$. Following this, the solution undergoes a local improvement step (Step 2), which will be discussed in the following subsection. In Step 1 of subsequent iterations $(i>1)$, the solution $\boldsymbol{x}^{r}$ is obtained from $\boldsymbol{x}^{r *}$ randomly modifying all the parameters of a controller (PSS or IPFC-POD). In this case, it can be seen as a step of disruption.

\subsection{Local Search Based on Sensitivities}

Assume that the solution $\boldsymbol{x}^{r}$ is obtained in Step 1 of the algorithm of Fig. 9. The evaluation function of $\boldsymbol{x}^{r}$ is defined as $F\left(\boldsymbol{x}^{r}\right)$, as shown in (43).

$$
\begin{aligned}
& F\left(\boldsymbol{x}^{r}\right)=f\left(\boldsymbol{x}^{r}\right)+\rho h\left(\boldsymbol{x}^{r}\right) \\
& f\left(\boldsymbol{x}^{r}\right)=\sum_{i=1}^{n}\left|\lambda_{i}^{\text {calc }}-\lambda_{i}^{\text {des }}\right| \\
& h\left(\boldsymbol{x}^{r}\right)=\sum_{i=1}^{n}\left|\xi_{i}^{\text {calc }}-\xi_{i}^{\text {des }}\right|
\end{aligned}
$$


In (43), the evaluation function $F\left(x^{r}\right)$, is composed of the objective function $f\left(\boldsymbol{x}^{r}\right)$, added to the infeasibility $h\left(\boldsymbol{x}^{r}\right)$. In addition, in (43), $\rho \gg 1$ is a penalty parameter and in (44) and (45) $n$ denotes the number of eigenvalues of interest. The objective function used to calculate the desired damping for a given proposed solution (considering the eigenvalues of interest) is defined as shown in (44). It minimizes the distance among the calculated eigenvalues of interest $\left(\lambda_{i}^{\text {calc }}\right)$ and the desired eigenvalues of interest $\left(\lambda_{i}^{\text {des }}\right)$, considering the appropriate limits (40)-(42). The desired eigenvalues $\left(\lambda_{i}^{\text {des }}\right)$ are defined by the system operator from the desired damping $\left(\xi_{i}^{\text {des }}\right)$ while the calculated eigenvalues of interest $\left(\lambda_{i}^{\text {calc }}\right)$ are determined by state matrix in the CSM at each iteration of the Multi-Start algorithm by adjusting the parameters of the controllers (PSSs and IPFC-POD).

A vector of sensitivity, $\boldsymbol{s}$, of dimension $n_{v}$ (where $n_{v}$ is the number of problem variables) is initially defined with all the elements, $s_{i}$, set to zero. The continuous variables are treated as a set of discrete variables, and the value of each discretization step for variable $i, \Delta_{i}$, is determined, as shown in (46), where $u_{i}$ and $l_{i}$ are the upper and lower bounds of the variable $x_{i}^{r}$, respectively, and $\Gamma$ is the integer number of the discretizations.

$$
\Delta_{i}=\left(u_{i}-l_{i}\right) / \Gamma \quad i=1, \cdots, n
$$

One problem variable is randomly chosen to modify the value. Suppose the variable $x_{k}^{r}$ is chosen. The value of the variation $\delta_{k}$ is determined as shown in (47), where $\varphi$ is a random number between zero and one $(0 \leq \varphi \leq 1), \gamma$ is a scaling factor and $[\cdot]$ is the ceiling function, which rounds the value of $\gamma \varphi$ up to the next whole number.

$$
\delta_{k}=\lceil\gamma \varphi\rceil \Delta_{k}
$$

The next step is to determine the new value of $x_{k}^{r}$, as shown in (48).

$$
\tilde{x}_{k}^{r}=x_{k}^{r} \pm \delta_{k}
$$

In (48), as initially $s_{k}=0$, an increase or decrease in the value of the variable $x_{k}^{r}$ is chosen with equal probability. Note that in (48), $\tilde{x}_{k}^{r}$ may infringe the lower limit, $l_{k}$, or the upper limit, $u_{k}$. In this case, consider the correction (49).

$$
\hat{x}_{k}^{r}= \begin{cases}\tilde{x}_{k}^{r}, & \text { if } l_{k} \leq \tilde{x}_{k}^{r} \leq u_{k} \\ l_{k}, & \text { if } \tilde{x}_{k}^{r}<l_{k} \\ u_{k}, & \text { if } \tilde{x}_{k}^{r}>u_{k}\end{cases}
$$

The evaluation function $F\left(\widehat{\boldsymbol{x}}^{r}\right)$ is then calculated for the new solution, and the quality of $\widehat{\boldsymbol{x}}^{r}$ is checked. If $F\left(\widehat{\boldsymbol{x}}^{r}\right) \leq F\left(\boldsymbol{x}^{r}\right)$, and in (48) $x_{k}^{r}$ was incremented, then $s_{k}=1$. If in (48), $x_{k}^{r}$ was decremented, then $s_{k}=-1$. Also, the current solution should be updated: $\boldsymbol{x}^{r} \leftarrow \widehat{\boldsymbol{x}}^{r}$. If $F\left(\widehat{\boldsymbol{x}}^{r}\right)>F\left(\boldsymbol{x}^{r}\right)$, then $s_{k}=-1$ if in (48) $x_{k}^{r}$ was incremented. If $x_{k}^{r}$ was decremented, then $s_{k}=1$. In this case, the current solution is not updated.

This process is performed until no improvement is obtained or until a maximum number of iterations without improvement, $\Upsilon$, is reached. The factor $\gamma$ should be adjusted dynamically during the process, that is, from $\tau$ iterations, $\tau<\Upsilon$, without improving the evaluation function, define $\gamma \leftarrow \gamma / 2$ in (47). $\rho=10^{3}, \Gamma=100$ and $\gamma=20$ were adopted. The pseudocode of the local search methodology is presented in Fig. 10.

\section{SIMULATIONS AND RESULTS}

The symmetrical system of two areas whose one-line diagram is shown in Fig. 11 Sauer and Pai (1998) has four generators, 17 transmission lines and 12 buses, with two new buses (buses BF1 and BF2), and two new transmission lines (lines L9 and L10) that are included solely to perform simulations with the IPFC. The values of the reactances of the coupling transformers $\left(x_{i n}, n=\right.$ $j, k)$ are stipulated at 0.01 p.u. For the PI controllers the following parameters were adopted: $T_{1_{i}}=0.50 \mathrm{~s}, T_{2_{i}}=0.10 \mathrm{~s}, T_{3_{i}}=0.10 \mathrm{~s}$, $T_{m_{p}}=0.005 \mathrm{~s}, K 1=0.80$ p.u., $K 2=0.50$ p.u. and $K 3=0.50$ p.u. The values, in p.u., of the variables that are used to represent the converters are small $\left(V_{p_{j}}=7.22 \times 10^{-6}, V_{p_{k}}=3.67 \times 10^{-5}, V_{q_{j}}=\right.$ $4.87 \times 10^{-5}$, and $V_{q_{k}}=6.52 \times 10^{-5}$ ). Thus, the IPFC is included in the EPS, but does not exercise any control over the active and reactive power flows.

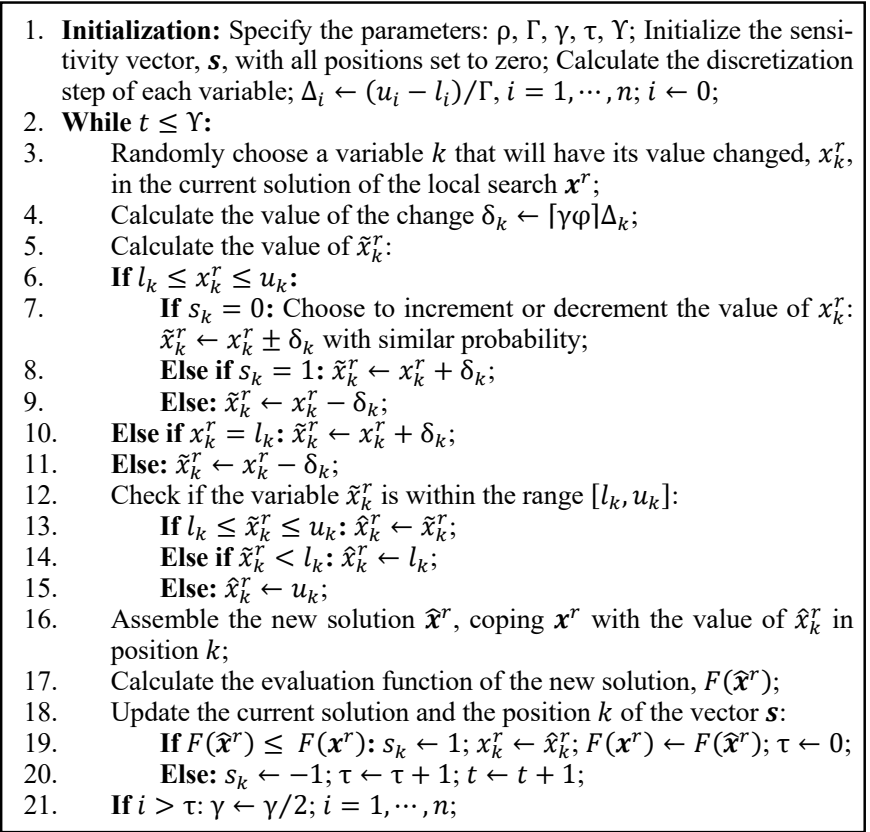

Figure 10. Local search.

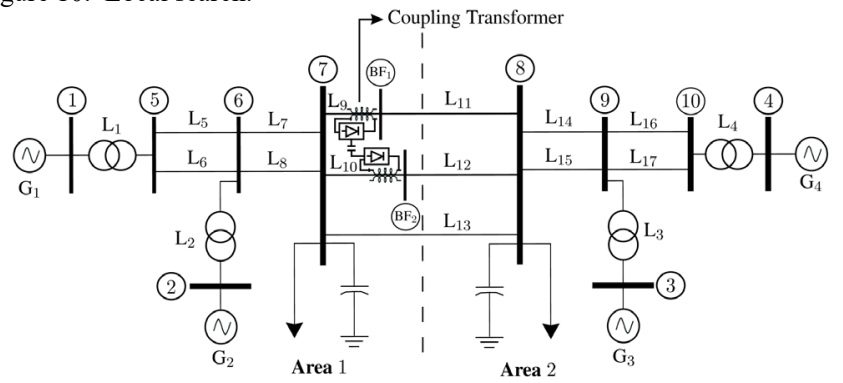

Figure 11. Diagram of the Two-Area System.

The voltage profile for the base case is shown in Fig. 12, which shows that the voltage at bus 7 is below 0.95 p.u. (0.94 p.u.). To improve the voltage at this bus, an IPFC was installed between buses 7 and 8 (see Fig. 11). After installing the device and controlling the active and reactive power flows, the voltage profile at bus 7 has changed (see Fig. 12). 
Note in Fig. 12 that the installation of the IPFC improves the voltage profile at bus 7 (0.9501 p.u.) to within the acceptable range ( $\pm 5 \%$ of the nominal value).

The flows of lines $L_{11}$ and $L_{12}$ were controlled by the IPFC for the results of Fig. 12 (see Fig. 11) with $P_{r e f_{j}}=75.83 \mathrm{MW}$ (base case: $64.81 \mathrm{MW}$ ), $Q_{r e f_{j}}=-79.87 \mathrm{MVAr}$ (base case: $-24.96 \mathrm{MW}$ ) and $P_{r e f_{k}}=75.83 \mathrm{MW}$ (base case: $64.81 \mathrm{MW}$ ). In this situation, the value of the variables used to represent the converters (in p.u.) are $V_{p_{j}}=0.1187, V_{p_{k}}=0.0545, V_{q_{j}}=-0.2406$, and $V_{q_{k}}=0.0964$.

The eigenvalues of the matrix of states of the test system were calculated for this configuration with the oscillation modes of interest $\left(\lambda_{i}=\sigma_{i} \pm j \omega_{i}\right)$, damping coefficients $\quad\left(\xi_{i}=\right.$ $-\sigma_{i} / \sqrt{\sigma_{i}{ }^{2}+\omega_{i}{ }^{2}}$ ) and natural undamped frequencies $\left(\omega_{n i}=\left|\lambda_{i}\right| / 2 \pi\right)$ being shown in Table 1 .

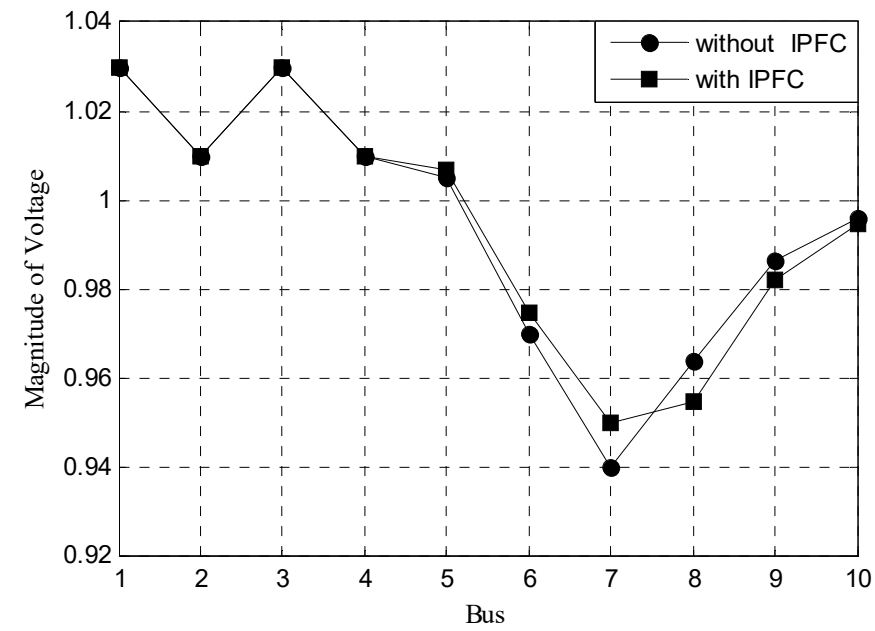

Figure 12. Profile of the bus voltages of the Two-Area System.

Table 1. Dominant Eigenvalues, Damping Coefficients and Natural Undamped Frequencies

\begin{tabular}{|c|c|c|c|}
\hline Mode & Eigenvalues & $\xi_{i}$ (p.u.) & Frequencies (Hz) \\
\hline$\lambda_{1}$ & $-0.3648 \pm \mathrm{j} 6.2987$ & 0.0578 & 1.0041 \\
\hline$\lambda_{2}$ & $-0.2621 \pm \mathrm{j} 5.9123$ & 0.0443 & 0.9419 \\
\hline$\lambda_{3}$ & $0.0526 \pm \mathrm{j} 4.4684$ & -0.0118 & 0.7112 \\
\hline
\end{tabular}

On analyzing the natural undamped frequencies of the eigenvalues shown in Table 1, the existence of two local modes $\left(\lambda_{1}\right.$ and $\left.\lambda_{2}\right)$ and an inter-area mode $\left(\lambda_{3}\right)$ with a positive real part are identified, which characterizes the instability of the EPS.

The purpose of this work is to install two PSSs and an IPFC-POD to provide damping to local and inter-area mode oscillations. Participation factors were used to define the location of the PSSs in the EPS Sauer and Pai (1998). Simulations (not presented in this paper) indicated that the best sites are Generators 2 and 3 Fortes et al. (2016c), thus, in this paper, the PSSs are installed at these generators.

After processing the Multi-Start algorithm, the parameters of the PSSs and IPFC-POD were determined while satisfying the constraints in (40)-(42). The values obtained are shown in Table 2 and this configuration should be able to impose the allocation of eigenvalues related to the local and inter-area modes so that their damping coefficients are in the range of $10 \%$ to $11 \%$.
Table 2. Gains and Time Constants of the PSSs and POD

\begin{tabular}{|c|c|c|c|}
\hline Parameters & PSS G2 & PSS G3 & IPFC-POD \\
\hline$T_{1}\left(T_{p_{1}}\right)=T_{3}\left(T_{p_{3}}\right)$ & 0.8100 & 0.7150 & 1.0000 \\
\hline$T_{2}\left(T_{p_{2}}\right)=T_{4}\left(T_{p_{4}}\right)$ & 0.6200 & 0.3350 & 0.9050 \\
\hline$K_{P S S}\left(K_{P O D}\right)$ & 2.2000 & 1.4000 & 0.7150 \\
\hline
\end{tabular}

The eigenvalues of the test system were calculated with the PSSs and IPFC-POD functioning in the EPS (adjusted with the parameters given in Table 2). The results are shown in Table 3.

Table 3. Dominant Eigenvalues, Damping Coefficients and Frequencies

\begin{tabular}{|c|c|c|c|}
\hline Mode & Eigenvalues & $\xi_{i}$ (p.u.) & Frequencies $(\mathrm{Hz})$ \\
\hline$\lambda_{1}$ & $-0.6504 \pm \mathrm{j} 6.3944$ & 0.1012 & 1.0230 \\
\hline$\lambda_{2}$ & $-0.6301 \pm \mathrm{j} 5.8946$ & 0.1063 & 0.9435 \\
\hline$\lambda_{3}$ & $-0.4722 \pm \mathrm{j} 4.3212$ & 0.1086 & 0.6918 \\
\hline
\end{tabular}

On analyzing Table 3 , it is concluded that the adjustment of the parameters of the controllers using the Multi-Start method reached the objective. Note that both the local modes and interarea mode are displaced into regions of the complex plane in such a way that their damping coefficients are between $10 \%$ and $11 \%$.

For analysis in the time domain, a disturbance of $5 \%$ in the mechanical power of Generator $4(\mathrm{G} 4)$, the reference generator of the test system, is considered. The angular variations of the first (G1), the second (G2), and third generators (G3) with respect to Generator 4 are shown in Fig. 13. In this simulation, the test system is equipped with two PSSs and an IPFC-POD adjusted using the parameters shown in Table 2.

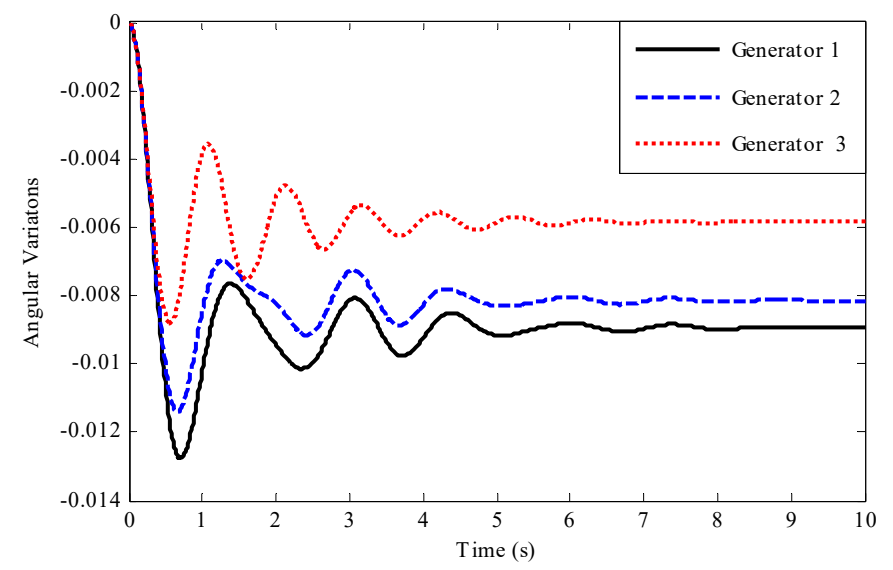

Figure 13. Angular variations of Generators G1, G2 and G3 with the parameters of Table 2 .

The curves shown indicate that, regardless of the physical area of the location of the generators, the oscillations are dampened, confirming the effectiveness of the Multi-Start algorithm in adjusting the damping of supplementary controllers.

\section{CONCLUSION}

An optimization technique based on the Multi-Start metaheuristic was used in this work to adjust the parameters of the supplementary damping controllers, PSSs and an IPFC-POD. The function of these controllers is to ensure the stability of the EPS in the event of a small disturbance. 
The EPS was represented by a linear model based on the nodal balance of currents. As a result, the FACTS IPFC was also modelled by current injection.

Considering the performance of the PSSs and the IPFC-POD in the EPS with the parameters being set by the Multi-Start algorithm, it was possible to make the test system stable for a given operating point. It was thus concluded that the algorithm is effective to adjust the parameters of controllers in order to re-allocate eigenvalues of interest in regions of the complex plane with damping coefficients stipulated in the design.

\section{ACKNOWLEDGEMENTS}

This work was supported by the Coordination for the Improvement of Higher Education Personnel (CAPES) - Finance Code 001 and the São Paulo Research Foundation (FAPESP), under grant 2018/20355-1.

\section{REFERENCES}

Anderson, P. and Fouad, A.A. (1993). Power system control and stability. WileyIEEE Press, New York.

De Mello, F.P. and Concordia, C. (1969). Concepts of synchronous machine stability as affected by excitation control. IEEE Transactions on Power Apparatus and Systems, PAS-88(4), 316-329.

Larsen, E. and Swann, D. (1981). Applying power system stabilizers Part II: Performance objectives and tuning concepts. IEEE Transactions on Power Delivery, PAS-100(6), 3025-3033.

Fortes, E.V., Araujo, P.B., and Macedo, L.H. (2016a). Coordinated tuning of the parameters of PI, PSS and POD controllers using a specialized chubeasley's genetic algorithm. Electric Power Systems Research, 140, 708721.

Fortes, E.V., Araujo, P.B., Macedo, L.H., and Gamino, B.R. (2016b). Ajuste coordenado dos parâmetros dos controladores ESP e IPFC-POD usando um algoritmo multi-start. In Congresso Brasileiro de Automática (CBA), 1-6. Vitória - ES

Fortes, E.V., Araujo, P.B., Macedo, L.H., Gamino, B.R., and Martins, L.F.B. (2016c). Analysis of the influence of PSS and IPFC-POD controllers in small-signal stability using a simulated annealing algorithm. In IEEE International Conference on Industry Applications (INDUSCON), 1-8. Curitiba - PR.

Fortes, E.V., Macedo, L.H., Araujo, P.B., and Romero, R. (2018). A VNS algorithm for the design of supplementary damping controllers for small-signal stability analysis. International Journal of Electrical Power \& Energy Systems, 94, 41-56.

Fortes, E.V., Macedo, L.H., Martins, L.F.B., and Miotto, E.L. (2017). A fireworks metaheuristic for the design of PSS and TCSC-POD controllers for smallsignal stability studies. In Latin American Congress on Generation, Transmission and Distribution (CLAGTEE), 1-6. Mar del Plata - AG.

Glover, F. and Kochenberger, G. (2003). Handbook of metaheuristics, volume 57 of International Series in Operations Research \& Management Scnce. Springer.

Gyugyi, L., Sen, K., and Schauderr, C.D. (1999). The interline power flow controller concept: a new approach to power flow management in transmission systems. IEEE Transactions on Power Delivery, 14(3), 1115- 1123.

Hingorani, N.G. and Gyugyi, L. (1999). Understanding FACTS. IEEE, New York.

Kundur, P. (1994). Power system stability and control. MacGraw-Hill, New York.

Martins, L.F.B., Araujo, P.B., Fortes, E.V., and Macedo, L.H. (2017). Design of the PI-UPFC-POD and PSS damping controllers using an artificial bee colony algo rithm. Journal of Control, Automation and Electrical Systems, 28, $1-12$.

Rezaei, N., Kalantar, M., Shayanfar, H., Alipouri, Y., and Safari, A. (2013). Optimal signal selection and damping controller design for IPFC using a novel current injection model in a multi-machine power system. International Journal of Electrical Power \& Energy Systems, 44, 461-470.

Sauer, P.W. and Pai, M.A. (1998). Power system dynamics and stability. Prentice Hall, New Jersey.
Takahashi, A.L.M., Fortes, E.V., Araujo, P.B., Miotto, E.L., and Martins, L.F.B. (2018). A current sensitivity model for power system stability studies. In $201813^{\text {th }}$ IEEE International Conference on Industry Applications (INDUSCON), 955-962. São Paulo - SP.

Valle, D. and Araujo, P. (2015). The influence of GUPFC FACTS device on small signal stability of the electrical power systems. International Journal of Electrical Power \& Energy Systems, 65, 299-306.

Wang, Z., Chung, C., Wong, K., Tse, C., and Wang, K. (2007). Robust PSS design under multioperating conditions using canonical particle swarm optimization. $1-7$.

Zhang, J. and Yokoyama, A. (2008). Power system transient stability improvent by the interline power flow controller (IPFC). IEEJ Transactions Power \& Energy, 128(1), 208-215.

Zhang, X.P. (2003). Modelling of the interline power flow controller and the generalised unified power flow controller in newton power flow. IEE Proceedings - Generation, Transmission and Distribution, 150(3), 268-274. 